\title{
THERMAL \\ A Routine Designed to Calculate Neutron Thermal Scattering
}

\author{
Dermott E. Cullen \\ Lawrence Livermore National Laboratory \\ L-294 \\ P.O. Box 808 \\ Livermore, CA 94551-0808
}

February 24, 1995

This is an informal report intended primarily for internal or limited external distribution. The opinions and conclusions stated are those of the author and may or may not be those of the Laboratory.

Work performed under the auspices of the U.S. Department of Energy by the Lawrence Livermore National Laboratory under Contract W-7405-Eng-48. 


\section{DISCLAIMER}

This document was prepared as an account of work sponsored by an agency of the United States Government. Neither the United States Government nor the University of California nor any of their employees, makes any warranty, express or implied, or assumes any legal liability or responsibility for the accuracy, completeness, orusefulness of any information, apparatus, product, or process disclosed, or nepresents that its use would not infringe privately owned rights. Reference herein to any specific commercial products, process, or service by trade name, trademark, manufacturer, or otherwise, does not necessarily constitute or imply its endorsement, recommendation, or favoring by the United States Government or the University of California. The views and opinions of authors expressed herein do not necessarily state or reflect those of the United States Government or the University of California, and shall not be used for advertising or product endorsement purposes.

This report has been reproduced directly from the best available copy.

Available to DOE and DOE contractors from the Office of Scientific and Technical Information P.O. Box 62, Oak Ridge, TN 37831

Prices available from (615) 576-8401, FTS 626-8401

Available to the public from the National Technical Information Service

U.S. Department of Commerce 5285 Port Royal Rd. Springfield, VA 22161 


\section{DISCLAIMER}

Portions of this document may be illegible in electronic image products. Images are produced from the best available original document. 


\title{
THERMAL: \\ A Routine Designed to Calculate Neutron Thermal Scattering
}

\author{
by \\ Dermott E. Cullen \\ Lawrence Livermore National Laboratory \\ L-294 \\ P.O. Box 808 \\ Livermore, CA 94550
}

February 241995

Introduction

THERMAL is designed to calculate neutron thermal scattering that is isotropic in the center of mass system. At low energy thermal motion will be included. At high energies the target nuclei are assumed to be stationary. The point of transition between low and high energies has been defined to insure a smooth transition.

It is assumed that at low energy the elastic cross section is constant in the center of mass system. At high energy the cross section can be of any form.

You can use this routine for all energies where the elastic scattering is isotropic in the center of mass system. In most materials this will be a fairly high energy.

\section{Documentation}

This routine is designed to be self-documenting, in the sense that the latest documentation for the routine is included as comment lines at the beginning of the routine. Reports, such as this one, are periodically published. However, the user should be aware that the comment lines within the routine are continuously updated to reflect the most recent status of the routine and these comment lines must always be considered the most recent documentation. Therefore the user is advised to always read the comment lines within the routine.

The remainder of this report consists of a listing of the documentation that appears at the beginning of the THERMAL routine and quality assurance results verifying the accuracy of this routine.

\section{Acknowledgments}

I thank Gene Canfield, (Lawrence Livermore National Laboratory) for contributing his time, energy and most of all his ideas concerning thermal scattering. 
Comments from THERMAL 


\title{
SUBROUTINE THERMAL(ATWT,TEMPE)
}

\author{
C \\ C NEUTRON THERMAL SCATTERING ROUTINE \\ C VERSION 95.1 (FEBRUARY 1995) \\ C \\ C Written by \\ C Dermott E. Cullen \\ C University of California \\ C Lawrence Livermore National Laboratory \\ C L-294 \\ C P.O. Box 808 \\ C Livermore, CA 94550 \\ C U.S.A. \\ C \\ C Tele: 415-423-7359 \\ C E. Mail: cullen1@llnl.gov \\ $\mathrm{C}$ \\ C PURPOSE \\ $\mathrm{C}$ \\ C Calculate elastic scattering that is isotropic in the center of \\ C mass system. At low energies thermal motion will be included. \\ C At high energies the target nuclei are assumed to be stationary. \\ C The point of transition between low and high energies has been \\ C defined to insure a smooth transition. \\ C It is assumed that at low energy the elastic cross section is \\ C constant. At high energy the cross section can be of any form. \\ $\mathrm{C}$ \\ C You can use this routine for all energies where the elastic \\ $\mathrm{C}$ scattering is isotropic in the center of mass system. In most \\ C materials this will be a fairly high energy. \\ C \\ C RELATED ROUTINES \\ $\mathrm{C}$ \\ C This routine is designed to be used in conjunction with the \\ C SIGMA1 method of Doppler broadening cross sections. It is assumed \\ $\mathrm{C}$ that the laboratory system cross sections you use in your Monte \\ C Carlo calculations have been pre-calculated using the SIGMA1 \\ C method. See, Ref. 1 \& 2 for SIGMA1 documentation. \\ C \\ C The approach used here is consistent with that used in the SIGMA1 \\ $\mathrm{C}$ method of Doppler broadening cross sections. If you have used the \\ C SIGMA1 method of Doppler broadening cross sections the reaction \\ $C$ rates that you obtain using this routine will be completely \\ C consistent. If you have not Doppler broadened your cross sections \\ $\mathrm{C}$ the low energy reaction rate calculated by this routine will be \\ C higher than those predicted by your cross sections. \\ C \\ C WARNING \\ C You must call THERM0 once to initialize parameters before you \\ C call this routine the first time - you need only call THERMO once.
}


$\mathrm{C}$

C $\quad$ ATWT $=$ Target atomic weight in neutron mass units

C TEMPE = Target temperature in MeV, e.g., $293 \mathrm{~K}=2.53 \mathrm{~d}-08 \mathrm{MeV}$

$\mathrm{C}$ VNOW $=$ Neutron speed in $\mathrm{cm} /$ shake (shake $=1.0-8$ seconds)

C ALPNOW $=$ Neutron direction cosine relative to $X$ axis

C $\quad B E T N O W=$ Neutron direction cosine relative to $Y$ axis

C GAMNOW $=$ Neutron direction cosine relative to $\mathrm{Z}$ axis

$\mathrm{C}$

$\mathrm{C}$

$\mathrm{C}$

C This routine passes ATWT and TEMPE as arguments and the remaining

C variables are passed through COMMON/WHERENOW/. If you wish to use

$\mathrm{C}$ a different convention you need merely add additional variables

$\mathrm{C}$ as arguments and delete COMMON/WHERENOW.

$\mathrm{C}$

$\mathrm{C}$

$\mathrm{C}$

C $\quad$ EROOM $=2.53 \mathrm{D}-08 \mathrm{MeV}$ (room temperature thermal energy)

C VROOM $=2.20 \mathrm{D}-03 \mathrm{~cm} /$ shake (room temperature thermal speed)

C VNMIN $=5.00 \mathrm{D}-04 \mathrm{~cm} /$ shake (minimum TART speed)

C VNMAX $=20.0$ - at higher incident speeds ignore thermal motion

C

$\mathrm{C}$

C

C

$\mathrm{C}$

C dividing line between the two treatments is controlled by (VNMAX),

C presently defined to be 20 . When the incident neutron speed is

C greater than (VNMAX) times the average thermalized neutron speed

C thermal motion is not included; otherwise it is included.

C

C (VNMAX) has been defined to insure a smooth transition between

C the high and low energy treatments. For (VNMAX) equal to 20, the

C incident energy must be 400 times the temperature of the medium,

C e.g., at room temperature of $0.0253 \mathrm{eV}$, thermal motion will be

$\mathrm{C}$ included up to about $10 \mathrm{eV}$. By this energy the average relative

C speed between neutron and target is within $0.1 \%$ of the neutron

C speed.

C

C

$\mathrm{C}$

C It is assumed that the low energy center of mass elastic

C scattering cross section is constant and that the target nuclei

C are distributed in an isotropic Maxwellian in the laboratory

C system, i.e., the so called free atom assumption.

C

C For light isotopes the elastic cross section is constant to

C fairly high energy, so that this assumption is valid to describe

$\mathrm{C}$ the important slowing down in moderators. For heavy isotopes

$\mathrm{C}$ this assumption may no longer be valid above the eV energy 
C range, but will be valid near thermal energies.

$\mathrm{C}$

C For a number of important moderators binding effects are very

$\mathrm{C}$ important, so that the free atom assumption is no longer valid.

C I recommend that you use the ENDF/B thermal scattering law data

$\mathrm{C}$ whenever possible. However, since thermal scattering law data is

C only available for a relatively small number of materials, in

C cases where this data is not available I recommend that you use

C this routine as a good approximation to thermalization.

ISOTROPIC VS. NON-ISOTROPIC ELASTIC SCATTERING

$\mathrm{C}$

C Isotropic elastic scattering in the center of mass system allows

C the calculations to be greatly simplified and sped up. In this

C case the contributions of the neutron and target to the motion

C of the center of mass remain fixed through the collision (as they

C always do) and the motion of the neutron in the center of mass

C becomes random in direction.

C

C In this case all we need do is randomize the center of mass

C direction of the neutron (randomly select 3 new direction cosines

C that are completely uncorrelated to the original direction cosines

C of the neutron) and then vectorially add it to the motion of

C the center of mass to define new laboratory neutron speed and

C directions; we do not have to worry about scattering through a

C given angular distribution, where the final direction cosines are

C correlated to the original direction cosines, and the subsequent

C transformation back to the laboratory system.

C

C This approach is valid at both high and low energies; the only

C difference between the two treatments being that at high energy

C the target is assumed to be stationary, so that its motion is not

C included in the vectorial addition used to define the new neutron

C speed and directions.

\section{RUNNING TIME}

C

C On a SPARC-10 the high energy treatment can sample 1 million

C histories in about 7 seconds, and the low energy treatment takes

C about 18 seconds; the high energy treatment is about 2-1/2 times

C faster than the low energy treatment.

C

\section{UNITS}

C

C As distributed this routine assumes energy in $\mathrm{MeV}$ and speed in

C $\mathrm{cm} /$ shake. Everything is normalized to the variables,

C $\quad \mathrm{EROOM}=2.53 \mathrm{D}-08 \mathrm{MeV}$ (room temperature thermal energy)

C VROOM $=2.20 \mathrm{D}-03 \mathrm{~cm} / \mathrm{shake}$ (room temperature thermal speed)

C VNMIN $=5.00 \mathrm{D}-04 \mathrm{~cm} / \mathrm{shake}$ (minimum TART speed)

$\mathrm{C}$

C To use any other units (e.g., $\mathrm{eV}$ and $\mathrm{cm} / \mathrm{second}$ ) all you need so is

C re-define these constants (see, ENTRY THERM0).

C

C MINIMUM ALLOWABLE SPEED 
C

C You do not want to accept speeds which are below the minimum

C energy of your neutron cross section data - any events that

$\mathrm{C}$ result in a lower speed will be rejected.

C

C See THERMO, which includes the minimum allowable speeds for users

$\mathrm{C}$ of the TART-175 group structure (minimum energy $1.307 \mathrm{D}-09 \mathrm{MeV}$ ),

C ENDL (minimum energy $1.0 \mathrm{D}-10 \mathrm{MeV}$ ) or ENDF/B (minimum energy

C $\quad 1.0 \mathrm{D}-11 \mathrm{MeV}$ ). If you are using one of these merely select the

C correct one in THERMO. If you are using some other data set you

C will have to either define the minimum in THERM0, or set the

C minimum in THERMO to 0.0 and handle these cases as they are

$\mathrm{C}$ returned to you by this routine. If you do nothing you will be

C using the TART minimum allowable speed by default.

$\mathrm{C}$

$\mathrm{C}$

C

C This routine treats everything in $\mathrm{REAL}^{*} 8=$ double precision on

C a SPARC-10, single precision on a CRAY. I recommend that you

C only use this routine in REAL*8 - otherwise CAVEAT EMPTOR!!!!

C

C

C

C

C

$\mathrm{C}$

C

C

C

C

$\mathrm{C}$

C

C

C

C

C

C

C

C

C

C

C

C

C

C

C

C

C

C

C The result is a sampling method that reproduces the integral

C of a Maxwellian to better than $0.0001 \%$ and the differential

C Maxwellian to better than $0.01 \%$ over the entire range. 
C The last step is to reject extremely low probability large

$C$ values of $Y$. In the distributed version of this routine the

$\mathrm{C}$ rejection is controlled by XMAX which is $=4$. The integral of

C a Maxwellian up to $X=4$ is $1-5.0 D-07$, i.e., the probability

$\mathrm{C}$ of sampling an $\mathrm{X}$ above 4 is $5.0 \mathrm{D}-07$, so that sampling efficiency

$\mathrm{C}$ is essentially $100 \%$.

C This is a conservation equation, where we want to define the Lab

C cross section, $\operatorname{Sig}(\mathrm{V})$, to conserve the reaction rate that is

C occurring in the center of mass system; it is important to

$\mathrm{C}$ understand we are conserving reaction rate, not cross section.

$\mathrm{C}$

C P(Vr) must be defined such that,

C

The starting point for deriving the above equation is,

$\mathrm{V} * \operatorname{Sig}(\mathrm{V})=\mathrm{INT}[-1,+1] \mathrm{d}(\mathrm{COS}) / 2 * \mathrm{INT}[\mathrm{Vt}: \mathrm{Vr}>0] \mathrm{Vr} * \operatorname{Sig}(\mathrm{Vr}) * \mathrm{P}(\mathrm{Vt}) \mathrm{d}(\mathrm{Vt})$

where the 2 integrals are over the target angular and energy

distributions. The only assumption is that the target distribution

is isotropic. We can rewrite this in the form,

$\mathrm{V}^{*} \operatorname{Sig}(\mathrm{V})=\mathrm{INT}[\mathrm{COS}] \mathrm{INT}[\mathrm{Vt}] \mathrm{Vr} * \operatorname{Sig}(\mathrm{Vr}) \mathrm{d}(\mathrm{COS}) / 2 * \mathrm{P}(\mathrm{Vt}) \mathrm{d}(\mathrm{Vt})$

In which case the proper $\mathrm{P}(\mathrm{Vr}) \mathrm{d}(\mathrm{Vr})$ to use is,

$\mathrm{d}(\mathrm{COS}) / 2 * \mathrm{P}(\mathrm{Vt}) \mathrm{d}(\mathrm{Vt})$

In the general case with an energy dependent cross section Sig(Vr) sampling this distribution will sample $\mathrm{Vr}^{*} \mathrm{Sig}(\mathrm{Vr})$ and it is very difficult to separate the two and define $\mathrm{Vr}$ and $\mathrm{Sig}(\mathrm{Vr})$. However, in the case of a constant cross section this distribution will sample Vr; based on the sampled target speed $\mathrm{Vt}$ and direction COS we can immediately define the relative speed $\mathrm{Vr}$ and direction,

C

$\mathrm{Vr}=\operatorname{Sqrt}[\mathrm{Vt} * * 2+\mathrm{V} * * 2+2 * \operatorname{COS} * \mathrm{~V} * \mathrm{Vt}]$

with no preferential treatment of any Vr. In this case there are no restrictions on the range of COS and Vt, i.e., we can sample any $\mathrm{Vt}$ and still be able to sample the entire range of COS such

$\mathrm{C}$ that we can always define a relative speed. 
C

C In other words independently sample the isotropic angular C distribution $\mathrm{d}(\mathrm{COS}) / 2$ and the distribution of target speeds

C $\quad \mathrm{P}(\mathrm{Vt}) \mathrm{d}(\mathrm{Vt})$ to define the relative speed, $\mathrm{Vr}$.

C

C Note, this is a general result which applies to any isotropic

C target distribution (isotropic, so that the angular and

C energy distributions are not correlated). Here I will assume

C $\mathrm{P}(\mathrm{Vt}) \mathrm{d}(\mathrm{Vt})$ is a Maxwellian, in which case we have the familiar

C Doppler broadening equation,

C

C

C

C

C

C

$\mathrm{C}$

C

C In this case all the Doppler broadening equation is doing is

C defining an average relative speed and the lab, Doppler

C broadened cross section is proportional to the ratio of the

C average relative speed $\langle\mathrm{Vr}\rangle$ to the incident neutron speed.

C

C At high energies (speeds) where the target speed becomes

C insignificant the relative speed approaches the neutron

C speed and the Doppler broadened cross section approaches

C a constant. At low energies (speeds) where the neutron speed

C becomes insignificant the relative speed approaches the

C average target speed (a constant) and the Doppler broadened

C will increase; at very low energies it will increase as $1 / \mathrm{V}$.

C

$\mathrm{C}$

C

$\mathrm{C}$

C

C Maxwellian $=4 / \mathrm{Sqrt}(\mathrm{Pi}) * \mathrm{~B} * 3 / 2 * \mathrm{Vt} * * 2 * \operatorname{Exp}(-\mathrm{B} * \mathrm{Vt} * * 2) * \mathrm{~d}(\mathrm{Vt})$

$\mathrm{C} \mathrm{B}=\mathrm{ATWT} /(2 * \mathrm{KT})$

C $\mathrm{X}=\operatorname{Sqrt}(\mathrm{B}) * \mathrm{Vt}$

$\mathrm{C} \mathrm{d}(\mathrm{X})=\mathrm{Sqrt}(\mathrm{B}) * \mathrm{~d}(\mathrm{Vt})$

C Maxwellian $=4 / \mathrm{Sqrt}(\mathrm{Pi}) * \mathrm{X}^{* * 2} * \operatorname{Exp}\left(-\mathrm{X}^{* * 2}\right) * \mathrm{~d}(\mathrm{X})$

C

C 1) Sample the target speed Vt from the Maxwellian.

$\mathrm{C}$

C 2) The target nuclei are isotropic in the lab system. Sample to

C define the target direction cosines (AT, BT, GT)

$\mathrm{C}$

C 3) The relative speed is,

C

C $\quad$ VRX $=$ ALPNOW $*$ VNOW $-A T * V T$

C VRY $=$ BETNOW*VNOW - BT*VT

C $\quad$ VRZ $=$ GAMNOW $*$ VNOW - GT*VT

C $\mathrm{VR}^{* *} 2=\mathrm{VRX}^{* *} 2+\mathrm{VRY}^{* *} 2+\mathrm{VRZ}^{* * 2}$ 
C

C 4) Isotropically scatter in the center of mass system. Randomly

C sample relative direction cosines (AS, BS, GS).

C

C In the center of mass system,

C $\quad \mathrm{VNCM}=\mathrm{VR} * \mathrm{ATWT} /(1+\mathrm{ATWT})=$ Center of mass neutron speed

C $\quad \mathrm{VCM}=\mathrm{VR} /(1+\mathrm{ATWT})=\mathrm{VR}-\mathrm{VNCM}=$ Center of mass speed

C

C Note, in the following equations the factor $1 /(1+\mathrm{ATWT})$ is

C omitted, except in the definition of the final neutron speed.

C

C 5) Vectorially add the center of mass, neutron and target speeds to

C define new components of the neutron speed,

C

$\mathrm{C} \quad \mathrm{VX}=[\mathrm{ALPNOW} * \mathrm{VNOW}+\mathrm{ATWT} *(\mathrm{AS} * \mathrm{VR}+\mathrm{AT} * \mathrm{VT})]$

C VY $\quad$ VY $Y^{\prime}$ BETNOW*VNOW + ATWT* $*$ BS*VR + BT*VT)]

C VZ' $\quad$ VGAMNOW*VNOW +ATWT*(GS*VR+GT*VT) $]$

C

C 6) The new neutron speed is,

$\mathrm{C}$

C VNOW' $\mathrm{VN}^{\prime}=\mathrm{Sqrt}\left[\mathrm{VX}^{\prime * *} 2+\mathrm{VY}^{\prime * *} 2+\mathrm{VZ}^{\prime * *} 2\right] /(1+\mathrm{ATWT})$

$\mathrm{C}$

$\mathrm{C}$ and the new direction cosines are,

C

C $\quad$ ALPNOW' $=V^{\prime} / \mathrm{SQRT}\left(\mathrm{VX}^{\prime * *} 2+\mathrm{VY}^{\prime * * 2}+\mathrm{VZ}^{\prime * * 2}\right)$

C $\quad$ BETNOW $^{\prime}=V^{\prime} Y^{\prime} / S Q R T\left(V X^{\prime * * 2}+V^{\prime} * * 2+V Z^{\prime * * 2}\right)$

C $\quad$ GAMNOW' $^{\prime}=\mathrm{VZ}^{\prime} / \mathrm{SQRT}\left(\mathrm{VX}^{\prime * *} 2+\mathrm{VY}^{\prime} * * 2+\mathrm{VZ}^{\prime * * 2}\right)$

$\mathrm{C}$

C 7) If VNOW' is less than the minimum acceptable speed (VNMIN),

$\mathrm{C}$ reject and start over at 1 )

C

C Otherwise return the sampled values.

C

C

C

C The target nuclei are assumed to be stationary. The treatment

C is a simplified version of the low speed treatment with,

C

C $\quad \mathrm{Vt}=0$

C $\quad \mathrm{Vr}=\mathrm{Vn}$

C

C 1) Isotropically scatter in the center of mass system. Randomly

C sample relative direction cosines (AS, BS, GS).

$\mathrm{C}$

C 2) Vectorially add the center of mass and neutron speeds to define

C new components of the neutron speed,

C

C $\mathrm{VX}^{\prime}=[\mathrm{ALPNOW}+\mathrm{ATWT} * \mathrm{AS}]$

$\mathrm{C} \quad \mathrm{VY}^{\prime}=[\mathrm{BETNOW}+\mathrm{ATWT} * \mathrm{BS}]$

C VZ' $\quad$ V $Z^{\prime}$ GAMNOW + ATWT $^{*}$ GS $]$

C

C 3) The new neutron speed is,

C

C VNOW' = VNOW*SQRT $\left[\mathrm{VX}^{\prime * *} 2+\mathrm{VY}^{\prime * *} 2+\mathrm{VZ}^{\prime * *} 2\right] /(1+\mathrm{ATWT})$ 
C

C and the new direction cosines are,

C ALPNOW' $=V^{\prime} / \mathrm{SQRT}\left(\mathrm{VX}^{\prime * * 2}+\mathrm{VY}^{\prime * *}+\mathrm{VZ}^{\prime * * 2}\right)$

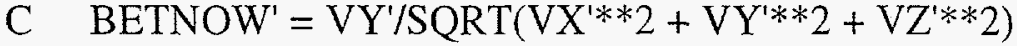

C GAMNOW' $=$ VZ'SQRT $\left(V^{\prime} X^{* * 2} 2+V^{\prime} * * 2+V Z^{* * 2}\right)$

C

C 4) If VNOW' is less than the minimum acceptable speed (VNMIN),

C reject and start over at 1 )

C

C Otherwise return the sampled values.

C

C

C

C If you are interested in the final target speed and direction,

C e.g., neutrons incident on hydrogen producing protons, the

$\mathrm{C}$ equations are very similar. In the center of mass system if

$\mathrm{C}$ after the collision the neutron is moving with direction cosines

$\mathrm{C}$ (+AS, $+\mathrm{BS},+\mathrm{GS})$, the target is moving with direction cosines

C $(-\mathrm{AS},-\mathrm{BS},-\mathrm{GS})$.

C Relative to the target the direction of the center of mass is

$\mathrm{C}$ the reverse of that relative to the neutron.

C

C All you need do is vectorially add the center of mass, neutron

$\mathrm{C}$ and target speeds to define the final target speed (VXT',

C VYT', VZT'), just as was done for the neutron to define (VX',

C VY', VZ') and then the above equations can be used to define

C the final target speed and directions.

C

C Starting from the above equations, exchange neutron and target,

$\mathrm{C}$ and reverse the center of mass directions,

C $\mathrm{VXT}^{\prime}=[\mathrm{AT} * \mathrm{VT}-\mathrm{ATWT} *(\mathrm{AS} * \mathrm{VR}+\mathrm{ALPNOW} * \mathrm{VNOW})]$

C $\mathrm{VYT}^{\prime}=\left[\mathrm{BT}^{*} \mathrm{VT}-\mathrm{ATWT} *(\mathrm{BS} * \mathrm{VR}+\mathrm{BETNOW} * \mathrm{VNOW})\right]$

C $\mathrm{VZT}^{\prime}=[\mathrm{GT} * \mathrm{VT}-\mathrm{ATWT} *(\mathrm{GS} * \mathrm{VR}+\mathrm{GAMNOW} * \mathrm{VNOW})]$

C

C That's the good news - the bad news is since this routine is

C restricted to isotropic scattering in the center of mass the

C energies involved will be relatively small and you will never

C have very energetic final targets - so this routine will be

C of little practical use to generate energetic targets, e.g.,

$\mathrm{C}$ for neutrons on hydrogen at these low energies you will never

C generate very energetic protons.

C REFERENCES

C

C 1) "Exact Doppler Broadening of Tabulated Cross Sections", by D. E. Cullen and

C C. R. Weisbin, Nuclear Science and Engineering: 60, 199-229 (1976)

C

C 2) "The 1994 ENDF Pre-processing codes", by D. E. Cullen, IAEA-NDS-39

C International Atomic Energy Agency (IAEA), Vienna, Austria, January 1994; this

C report includes the latest documentation for the SIGMA1 code.

$\mathrm{C}$ 


\section{Quality Assurance}

This routine is distributed with a driver program TESTER that allows users to verify correct implementation of THERMAL and to test each step in the calculations. For those users who have the PLOTTAB code results can be immediately viewed (for a copy of PLOTTAB contact the author).

Here TESTER will be used to verify the results of each step of the calculations. The steps that are verified include,

1) Sampling a Maxwellian

2) Sampling relative speed

3) Calculation of average relative speed

4) Collision by collision thermalization 


\section{Sampling a Maxwellian}

In dimensionless form a Maxwellian is,

$4 / \mathrm{Sqrt}(\mathrm{pi}) * \mathrm{X}^{* *} 2 * \operatorname{Exp}(-\mathrm{X} * * 2) * \mathrm{~d}(\mathrm{X})$

$\mathrm{X}=\mathrm{Sqrt}(\mathrm{b}) * \mathrm{Vt}$

$\mathrm{b}=\mathrm{ATWT} / 2 \mathrm{KT}$

The next figure compares an exact Maxwellian to the results obtained randomly sampling using the THERMAL algorithm. When methodically sampled the THERMAL algorithm reproduces the integral of a Maxwellian to within $0.0001 \%$ and the differential to within $0.01 \%$ over the entire range of X. Even when randomly sampled the next figure illustrates the excellent agreement. On the left hand side of this figure the agreement is so good that it is hard to tell that there are two separate curves. On the right hand side of the figure we can see that across the important center of the Maxwellian even random sampling produces agreement to within about $0.1 \%$.

Bottom line: the results verify that the THERMAL algorithm can accurately sample a Maxwellian. 

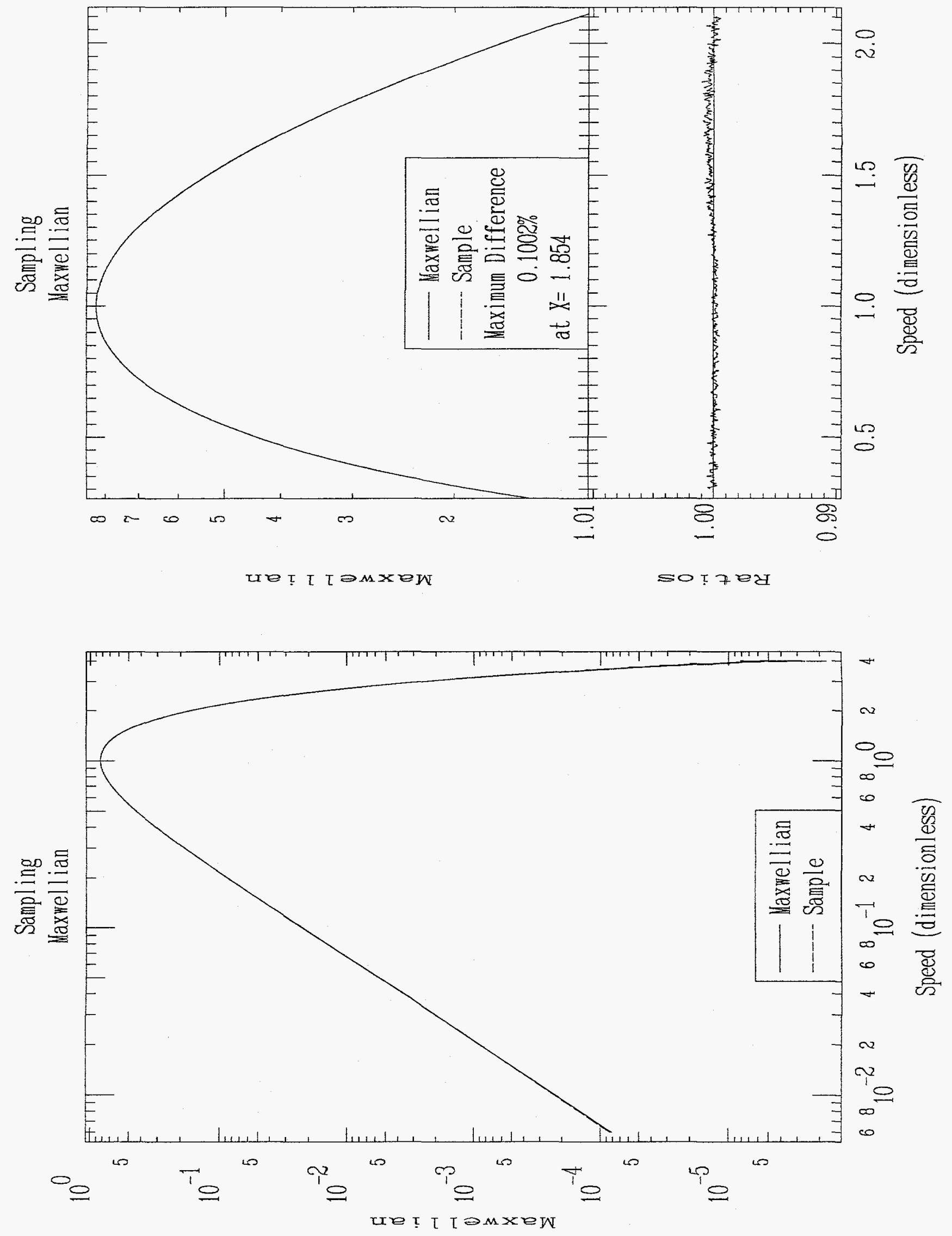


\section{Sampling Relative Speed}

In dimensionless form the distribution of relative speeds is,

$1 / \operatorname{Sqrt}(\mathrm{pi}) * \mathrm{Y} *\{\operatorname{Exp}[-(\mathrm{Y}-\mathrm{X}) * * 2]-\operatorname{Exp}[-(\mathrm{Y}+\mathrm{X}) * * 2]\} * \mathrm{~d}(\mathrm{Y}) / \mathrm{X}$

$\mathrm{X}=\mathrm{Sqrt}(\mathrm{b}) * \mathrm{Vn}-\mathrm{Neutron}$ laboratory speed

$\mathrm{Y}=\mathrm{Sqrt}(\mathrm{b})^{*} \mathrm{Vr}-$ Relative speed between neutron and target

$\mathrm{b}=\mathrm{ATWT} / 2 \mathrm{KT}$

It is important to note I am not directly sampling this distribution. THERMAL samples a Maxwellian to define target speed, $\mathrm{Vt}$, and an isotropic angular distribution to define the target direction cosines. The relative speed is then defined as,

$\mathrm{Vr}=\mathrm{Sqrt}\left[\mathrm{Vt} * * 2+\mathrm{Vn}^{* * 2}+2 * \operatorname{COSVt} * \mathrm{Vt} * \mathrm{Vn}\right]$

Therefore a critical step in quality assurance is to verify that the sampling method used by THERMAL actually reproduces the above distribution of relative speeds.

The next three figures compare the exact distributions to the results obtained randomly sampling a Maxwellian to define target speed and an isotropic distribution.

The first figure includes results for small values of $X=0.001,0.01,0.1$ and 1 ; neutrons that are extremely sub-thermal up to thermal energy. Again the agreement is so good that it is difficult to tell there are two curves on each plot, except at the high end of the distribution where the probability is extremely low, so that not too many samples occur = poor statistics.

The next figure includes results for $\mathrm{X}=2,3,4$ and 5. Compared to the previous low $\mathrm{X}$ results, in this $X$ range the shape of the distribution is changing as the effect of the second exponential, $\operatorname{Exp}\left[-(\mathrm{Y}+\mathrm{X})^{* * 2}\right.$ ], is becoming progressively smaller and eventually becomes insignificant.

The next figure includes results for $X=6,7,8$ and 10. Compared to the previous results by this $\mathrm{X}$ range the second exponential is completely insignificant and there is no longer any significant low energy "tail" to the distribution. Note the agreement over the entire range of relative speeds, except at the low and high ends of the distribution where the probability is extremely low, so that not too many samples occur $=$ poor statistics.

At higher values of $X$ the distribution is essentially,

$1 / \mathrm{Sqrt}(\mathrm{pi}) *(\mathrm{Y} / \mathrm{X}) * \operatorname{Exp}[-(\mathrm{Y}-\mathrm{X}) * * 2] * \mathrm{~d}(\mathrm{Y})$

and at very high $X, Y$ will be sampled only in a very narrow range about $X$, i.e., the relative speed $(\mathrm{Y})$ approaches the neutron speed $(\mathrm{X})$. So that at very high $\mathrm{X}$ a good approximation that is often used is that $(\mathrm{Y} / \mathrm{X}) \sim 1$ and the distribution becomes,

$1 / \operatorname{Sqrt}(\mathrm{pi}) * \operatorname{Exp}[-(\mathrm{Y}-\mathrm{X}) * * 2] * \mathrm{~d}(\mathrm{Y})$

an error function, Erf, symmetric about X.

Bottom line: the results verify that the THERMAL algorithm can accurately sample the distribution of relative speeds. 

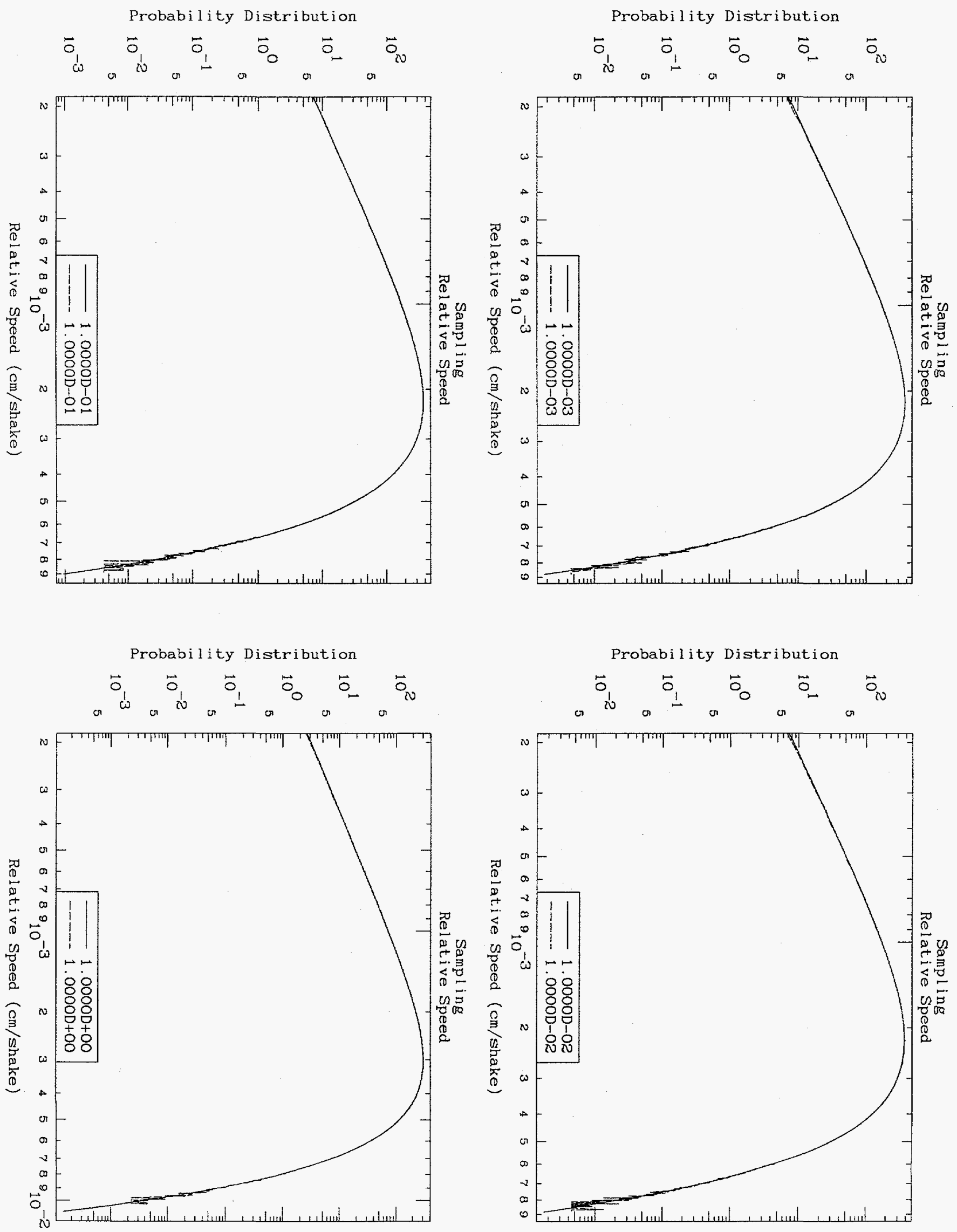
Probability Distribution
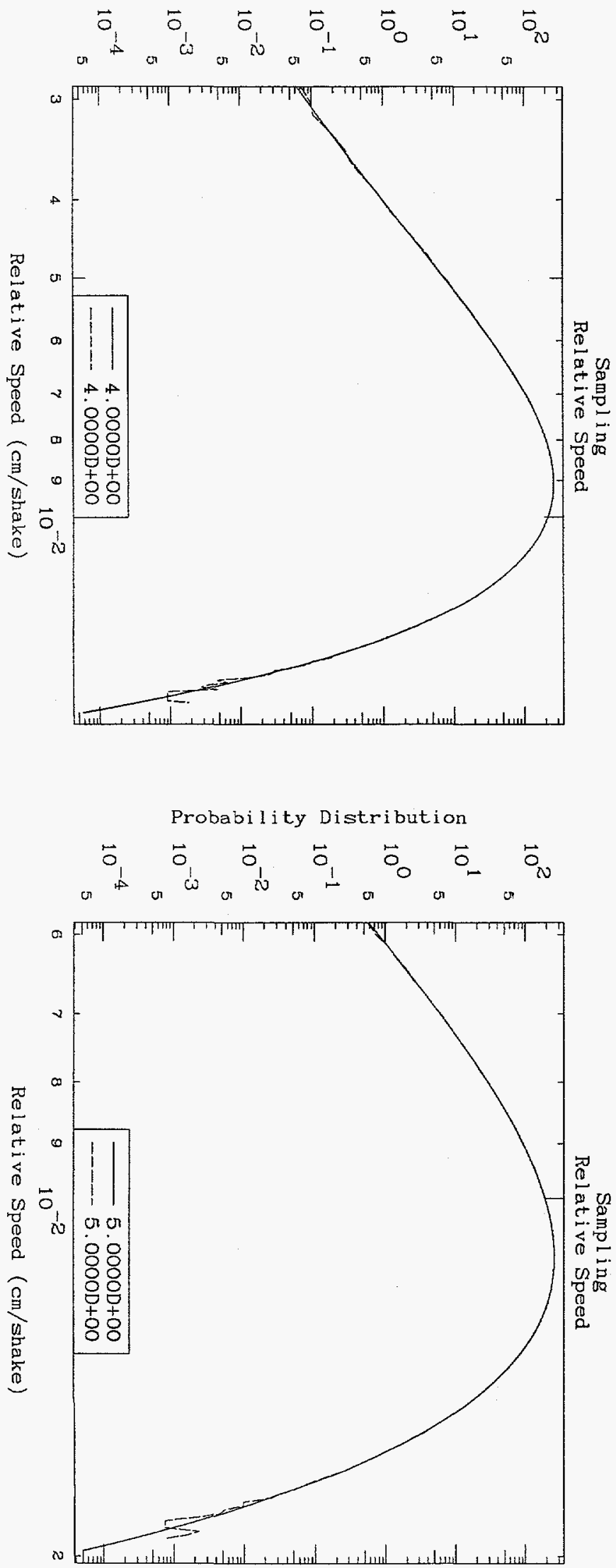

Probability Distribution
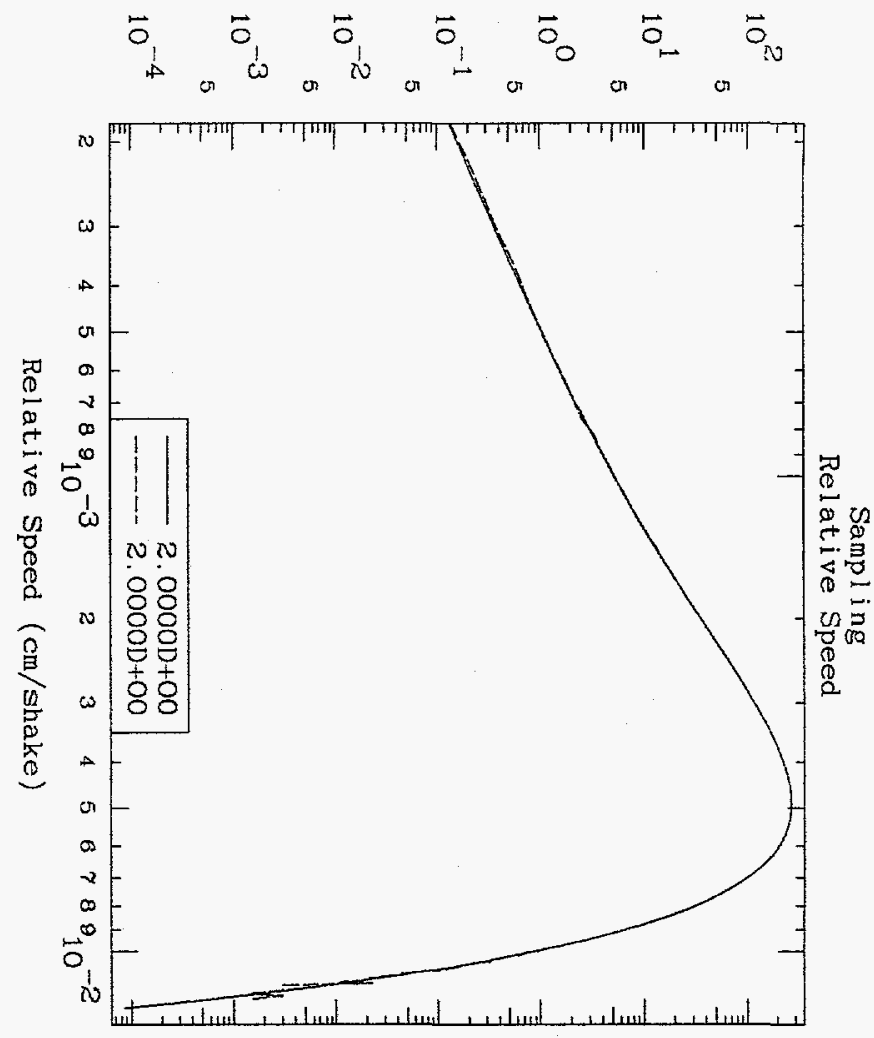

Probability Distribution
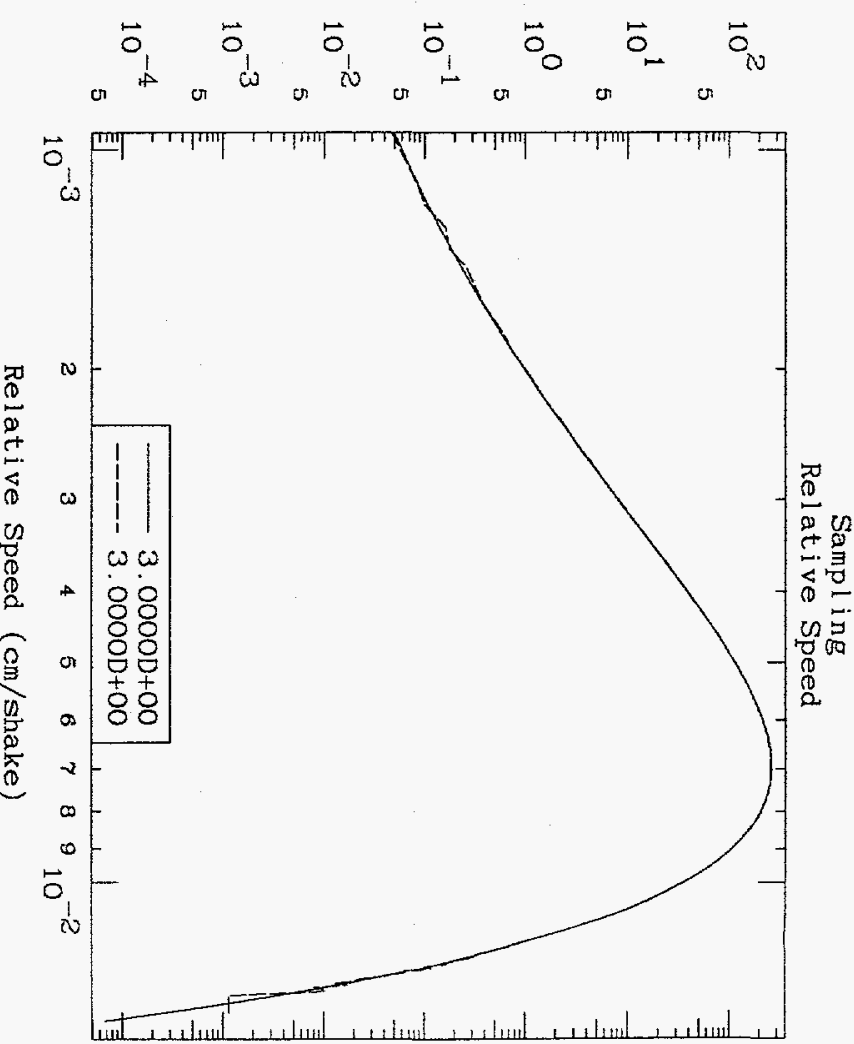

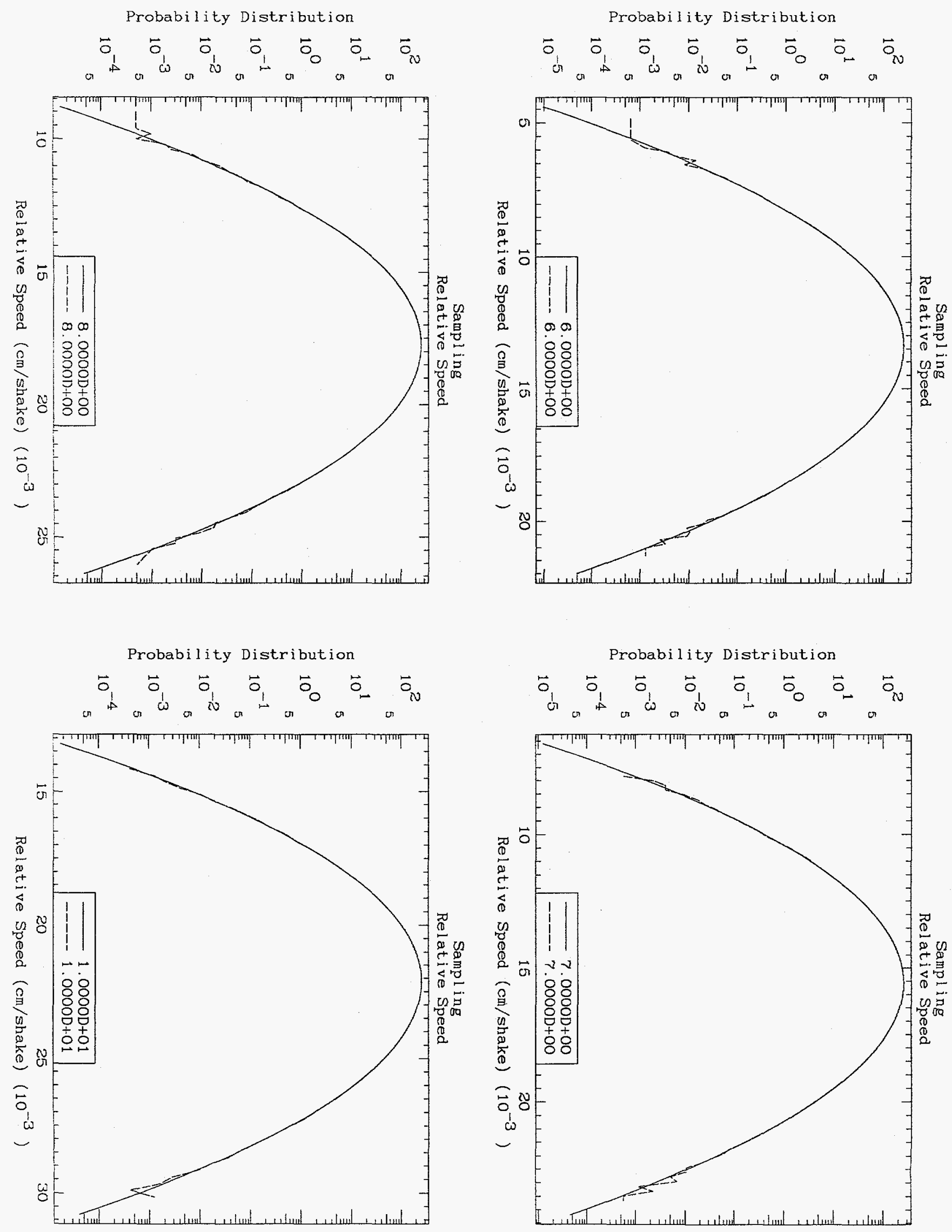


\section{Calculation of Average Relative Speed}

On the basis of the preceding results where it was verified that THERMAL can accurately sample the distribution of relative speeds, one could assume that it can also calculate the average relative speed. However, because of the importance of this quantity this will not be assumed - it will be verified.

In dimensionless form in the case of an elastic scattering cross section that is constant in the center of mass system the Doppler broadening equation can be written in the form,

$\mathrm{X} * \operatorname{Sig}(\mathrm{X})=\operatorname{Sigcm}^{*}<\mathrm{Y} * \mathrm{P}(\mathrm{Y}) * \mathrm{~d}(\mathrm{Y})><\mathrm{P}(\mathrm{Y}) * \mathrm{~d}(\mathrm{Y})>$

$\mathrm{P}(\mathrm{Y})^{*} \mathrm{~d}(\mathrm{Y})=1 / \mathrm{Sqrt}(\mathrm{pi}) * \mathrm{Y} *\left\{\operatorname{Exp}\left[-(\mathrm{Y}-\mathrm{X})^{* * 2}\right]-\operatorname{Exp}\left[-(\mathrm{Y}+\mathrm{X})^{* * 2}\right]\right\} * \mathrm{~d}(\mathrm{Y}) / \mathrm{X}$

$\mathrm{X}=\operatorname{Sqrt}(\mathrm{b}) * \mathrm{Vn}-$ Neutron laboratory speed

$\mathrm{Y}=\mathrm{Sqrt}(\mathrm{b}) * \mathrm{Vr}-$ Relative speed between neutron and target

$\mathrm{b}=\mathrm{ATWT} / 2 \mathrm{KT}$

The solution is,

$\mathrm{X} * \operatorname{Sig}(\mathrm{X})=\operatorname{Sigcm} *\left\{[\mathrm{X}+1 /(2 * \mathrm{X})] * \operatorname{Erf}(\mathrm{X})+\operatorname{Exp}\left(-\mathrm{X}^{* * 2}\right) / \operatorname{Sqrt}(\mathrm{pi})\right\}$

In this case all the Doppler broadening equation is doing is doing is defining an average value of the relative speed, $\langle\mathrm{Y}\rangle$. The laboratory, Doppler broadened cross section, $\operatorname{Sig}(\mathrm{X})$, is then,

$\operatorname{Sig}(\mathrm{X})=\operatorname{Sigcm}^{*}<\mathrm{Y}>/ \mathrm{X}$

That's why it is important to verify that THERMAL can correctly calculate the average relative speed, $\langle Y\rangle$. At low incident neutron speeds, $X,\langle Y\rangle$ will approach a constant, as the neutron speed becomes insignificant and the relative speed approaches the average target speed $=2 /$ Sqrt $($ pi) times Sqrt(KT/AE). So that the low energy Doppler broadened cross section approaches $C / X$,i.e., a $1 / \mathrm{N}$ cross section. At high incident neutron speeds, $\mathrm{X},\langle\mathrm{Y}>$ will approach $\mathrm{X}$, as the target speed becomes insignificant and the relative speed approaches the neutron speed. At higher energies this approach to equality can be written,

$<\mathrm{Y}>\sim \mathrm{X}+1 /(2 * \mathrm{X})$

and the Doppler broadened cross section approaches a constant.

The following table presents results for a wide range of incident neutron energies. In each case for a fixed incident neutron energy results were randomly sampled. In this case I used ATWT $=1$ (hydrogen) at room temperature $(293 \mathrm{~K})$, for incident neutron speed between $1 / 1000$ and 100 times thermal speed $(2200$ meters $/$ second $=2.2 \mathrm{D}-03 \mathrm{~cm} / \mathrm{shake}$ ). 
Results include

1) $\mathrm{AE} / \mathrm{KT} \quad \sim \mathrm{X}^{* * 2}$ - the common form for presenting results.

2) $\left\langle V^{*}\right\rangle \quad$ - average neutron speed after collision.

3) $<\mathrm{Vr}\rangle \quad$ - average relative speed.

4) $<\mathrm{Vt}\rangle \quad$ - average target speed.

5) $<\mathrm{Vr}>\mathrm{Vn} \quad$ - the Doppler broadened cross section per barn of constant center of mass cross section.

Both sampled and exact results are presented; see Ref. 1 for derivation of exact results.

6) $\%(\mathrm{~S}-\mathrm{E}) / \mathrm{E} \quad$ - the per-cent difference between the sampled and exact results.

7) Sec. - - running time in seconds (per million samples)

In this case the results that we expect are,

1) $\langle\mathrm{Vt}\rangle \quad-$ the Maxwellian averaged speed $=2 /$ Sqrt(pi) times thermal speed $=$ $2.482 \mathrm{~d}-3 \mathrm{~cm} / \mathrm{shake}$

2) $\langle\mathrm{Vr}\rangle \quad$ - at low energy it approaches $\langle\mathrm{Vt}\rangle$ and at high energy it approaches $\mathrm{Vn}$.

3) $\%(\mathrm{~S}-\mathrm{E}) / \mathrm{E}$ - the differences indicate that THERMAL can reproduce the correct average relative speed over the entire range to within about $0.1 \%$ or better. Note, the dividing line between the low high energy treatments where the times drops from 20 and 8 seconds - note that the high energy treatment still reproduces the correct relative speed to within $0.1 \%$.

Bottom line: the results verify that the THERMAL algorithm can accurately reproduce the correct average relative speed, which is equivalent to producing the correct Doppler broadened cross section. 
$\begin{array}{llllll}1.00 D-06 & 1.6551 D-03 & 2.4827 D-03 & 2.4827 D-03 & 1.1285 \mathrm{D}+03 & 1.1284 \mathrm{D}+03\end{array}$ $\begin{array}{lllllll}1.58 D-06 & 1.6561 D-03 & 2.4838 D-03 & 2.4838 D-03 & 8.9681 D+02 & 8.9631 D+02\end{array}$

0.011

0.057

0.050

0.032

0.053

0.015

0.020

0.088

0.041

$-0.020$

0.043

0.113

0.030

0.058

$-0.064$

0.025

0.018

0.065

$-0.084$

0.048

0.032

$-0.005$

$-0.010$

0.005

$-0.024$

0.067

$-0.007$

0.021

0.030

$-0.008$

0.024

0.046

$-0.002$

$-0.010$

$-0.016$

$-0.013$

0.000

0.008

$-0.001$

$-0.019$

0.002

0.008

0.005

0.003

$-0.079$

$-0.050$

$-0.032$

$-0.020$

$-0.013$

$-0.008$

$-0.005$
19.8

19.8

19.8

19.8

19.8

19.8

19.8

19.8

19.8

19.8

20.3

19.7

19.7

19.8

19.8

19.8

19.8

19.8

19.7

19.7

19.7

19.7

19.7

19.8

19.7

19.7

19.7

19.7

19.8

19.7

19.7

19.7

19.7

19.7

19.7

19.7

19.7

19.7

19.8

19.8

19.7

20.2

19.8

19.7

8.4

8.3

8. 4

8.3

8.3

8.4

8.4 


\section{Collision by Collision Thermalization}

Finally as the last step in verification we come to the proof of the pudding: does this puppy correctly thermalize neutrons? In order to verify that it does I have considered an idealized case with only constant, isotropic in the center of mass, elastic scattering and I follow 1 million individual histories as they slow down and hopefully correctly thermalize. In this idealized case with no absorption we expect them to slow down into a Maxwellian and stay there forever.

The following table presents collision by collision results for neutrons initially at an energy of $100 \mathrm{KT}$ (a speed 10 times thermal). The table included collision number, 1,2, 3, etc., time per collision in seconds, and a comparison between the exact first four moments of the Maxwellian and the sampled moments. If the neutrons correctly slow down we expect that after a number of collisions the neutrons will thermalize and the sampled moments should approach the exact moments. It is also important to verify that the neutrons will then stay in the Maxwellian forever, or to be practical, at least for a significant number of collisions.

The following table illustrates that after about 20 collisions the neutrons have thermalized; note, that within statistics the sampled and exact moments agree. The table also illustrates that the neutrons stays in the Maxwellian; note, after 40 collisions the sampled and exact moments still agree.

The following figure presents the collision by collision results for this case compared to a Maxwellian. Each curve represents the distribution of the 1 million histories after each has undergone the same number of collisions, 1,2,3, etc. The upper, left hand plots shows the first 10 collisions, where we can see the neutrons starting 10 times above thermal speed and progressively slowing down through each successive collision. The upper, right hand plot shows collisions 11 through 20 ; by 20 the distribution is essentially a Maxwellian. The lower, left plot shows collisions 21 through 30 , and finally the lower, right plot shows the collisions 31 through 40 over the important part of the Maxwellian. The ratio of an exact Maxwellian to the sampled results indicates statistical noise because of the sample size, but otherwise show excellent agreement with no bias at all, i.e., we see the same noise all the way across the plot with no bias toward left, center or right.

Note, that the distribution does not extend all the way down to zero speed. This is because of the low speed rejection, to only sample neutron speeds that are not below the lower limit of our neutron cross sections. In this case I have used the ENDF/B lower limit of 1.0d-05 $\mathrm{eV}(1.0 \mathrm{~d}-11 \mathrm{MeV})$.

The next figure presents similar results for slowing down in U-238. In this case many more collisions are required to thermalize the neutrons, but once again we see that they do eventually thermalize and stay there. The lower, right plot once again shows excellent agreement with no bias at all.

The final figure illustrates the high and low energy treatments. In this case we have initially $2.5 \mathrm{MeV}$ neutrons slowing down in hydrogen. After about 40 collisions they have all thermalized.

Bottom line: the results verify that the THERMAL algorithm can accurately thermalize neutrons and that once thermalized they will stay in the same thermalized distribution forever as long as there no absorption; naturally in any real system they would be removed by absorption. 
$\left\langle V_{n} * * 2>\right.$

$\langle\mathrm{Vn} * * 3\rangle$

1. $5000 D+00$

$2.2568 \mathrm{D}+00$

Sampled

$6.7291 D+00$

21.66 Seconds

Exact

Sampled

3

21.57 Seconds

Exact

Sampled

21.54 Seconds

Exact

1. $1284 \mathrm{D}+00$

4. 5944D+00

$\langle\mathrm{V} n$ >

1. $1284 \mathrm{D}+00$

3. $2234 \mathrm{D}+00$

$\langle\mathrm{Vn}\rangle$

1. $1284 \mathrm{D}+00$

Sampled

\section{60 Seconds} Exact Sampled

21.59 Seconds Exact Sampled

21. 60 Seconds Exact Sampled

$5.0742 D+01$

$\left\langle\mathrm{Vn} *{ }^{2}\right\rangle$

1. $5000 \mathrm{D}+00$

2. $6134 \mathrm{D}+01$

$\left\langle\mathrm{Vn}^{2} * 2\right\rangle$

1. $5000 \mathrm{D}+00$

1. $3828 \mathrm{D}+01$

$\langle\mathrm{Vn} \star \star 2\rangle$

1. $5000 \mathrm{D}+00$

$7.6668 \mathrm{D}+00$

$4.0799 \mathrm{D}+02$ $\langle\mathrm{Vn} * * 3\rangle$

$2.2568 \mathrm{D}+00$

1. $6878 \mathrm{D}+02$

$\langle\mathrm{Vn} * * 3\rangle$

$2.2568 \mathrm{D}+00$

7.1456D+01 $\langle\mathrm{Vn} * * 3\rangle$

$2.2568 \mathrm{D}+00$

3. $1436 \mathrm{D}+01$
$\left\langle\mathrm{Vn}_{n} * 4\right\rangle$

3. $7500 \mathrm{D}+00$

3. $4171 \mathrm{D}+03$

$\left\langle\mathrm{Vn}^{\star \star} \star 4>\right.$

3. $7500 \mathrm{D}+00$

1. $1831 \mathrm{D}+03$

$<\mathrm{Vn} * \star 4>$

3. $7500 \mathrm{D}+00$

4. $1792 \mathrm{D}+02$ $\left\langle\mathrm{Vn}^{*} * 4>\right.$

3. $7500 \mathrm{D}+00$

1. $5222 \mathrm{D}+02$ Exact Sampled

21.61 Seconds Exact Sampled

21.67 Seconds Exact Sampled

21.66 Seconds Exact Sampled

$\langle\mathrm{Vn}>$

1. $1284 \mathrm{D}+00$

1. $1282 \mathrm{D}+00$

<Vn>

1. $1284 \mathrm{D}+00$

1. $1286 \mathrm{D}+00$

$<\mathrm{Vn}>$

1. $1284 \mathrm{D}+00$

1. $1284 \mathrm{D}+00$
$<\mathrm{Vn} * * 2>$

$1.5000 \mathrm{D}+00$

1. 4993D+00

$<\mathrm{Vn} * \star 2>$

1. $5000 \mathrm{D}+00$

1. 4998D+00 $<\mathrm{Vn} \star \star 2>$

1. $5000 \mathrm{D}+00$

$1.4999 \mathrm{D}+00$
$<\mathrm{Vn} * \star 3>$

$2.2568 \mathrm{D}+00$

$2.2547 \mathrm{D}+00$

$\langle\mathrm{Vn} * * 3\rangle$

$2.2568 \mathrm{D}+00$

$2.2548 \mathrm{D}+00$ $<\mathrm{Vn} * * 3>$

$2.2568 \mathrm{D}+00$

$2.2567 \mathrm{D}+00$
$\langle\mathrm{Vn} * \star 4>$

3. $7500 D+00$

$3.7438 \mathrm{D}+00$ $<\mathrm{Vn} * * 4>$

3. $7500 \mathrm{D}+00$

3. $7425 \mathrm{D}+00$ $<\mathrm{Vn} * * 4>$

3. $7500 \mathrm{D}+00$

3. $7502 \mathrm{D}+00$

Sampled

\section{$<\mathrm{Vn}>$}

1. $1284 \mathrm{D}+00$

$1.1281 D+00$

<Vn>

1. 1284D+00

1. $1286 \mathrm{D}+00$

<Vn>

1. $1284 \mathrm{D}+00$

1. $1290 \mathrm{D}+00$

$<\mathrm{Vn}>$

1. $1284 \mathrm{D}+00$

$1.1285 \mathrm{D}+00$

$<\mathrm{Vn}\rangle$

1. $1284 \mathrm{D}+00$

1. $1292 \mathrm{D}+00$
$\langle\mathrm{Vn} * \star 2>$

1. $5000 \mathrm{D}+00$

1. $4984 \mathrm{D}+00$

$\langle\mathrm{Vn} * \star 2\rangle$

1. $5000 \mathrm{D}+00$

$1.5001 D+00$

$<\mathrm{Vn} * \star 2>$

1. $5000 \mathrm{D}+00$

1. $5009 \mathrm{D}+00$ $<\mathrm{Vn} * * 2>$

1. $5000 \mathrm{D}+00$

1. $4999 \mathrm{D}+00$

$<\mathrm{Vn} * * 2>$

1. $5000 \mathrm{D}+00$

$1.5020 \mathrm{D}+00$
$\langle\mathrm{Vn} * * 3\rangle$

2. $2568 \mathrm{D}+00$

$2.2516 \mathrm{D}+00$

$\langle\mathrm{Vn} * * 3\rangle$

$2.2568 \mathrm{D}+00$

$2.2561 D+00$ $\langle\mathrm{Vn} * \star 3>$

$2.2568 \mathrm{D}+00$

$2.2580 \mathrm{D}+00$ $<\mathrm{Vn} * * 3>$

2. $2568 \mathrm{D}+00$

$2.2559 \mathrm{D}+00$ $<\mathrm{Vn} * * 3>$

2. $2568 \mathrm{D}+00$

2. $2612 \mathrm{D}+00$
$<\mathrm{Vn} * * 4>$

3. $7500 \mathrm{D}+00$

3. $7353 \mathrm{D}+00$

$<\mathrm{Vn} * * 4>$

3. $7500 \mathrm{D}+00$

3. $7471 D+00$ $\langle\mathrm{Vn} * * 4\rangle$

3. $7500 \mathrm{D}+00$

3. $7515 \mathrm{D}+00$ $<\mathrm{Vn} * * 4>$

3. $7500 \mathrm{D}+00$

3. $7465 \mathrm{D}+00$

$\langle\mathrm{Vn} * * 4>$

3. $7500 \mathrm{D}+00$

3. $7601 \mathrm{D}+00$ 

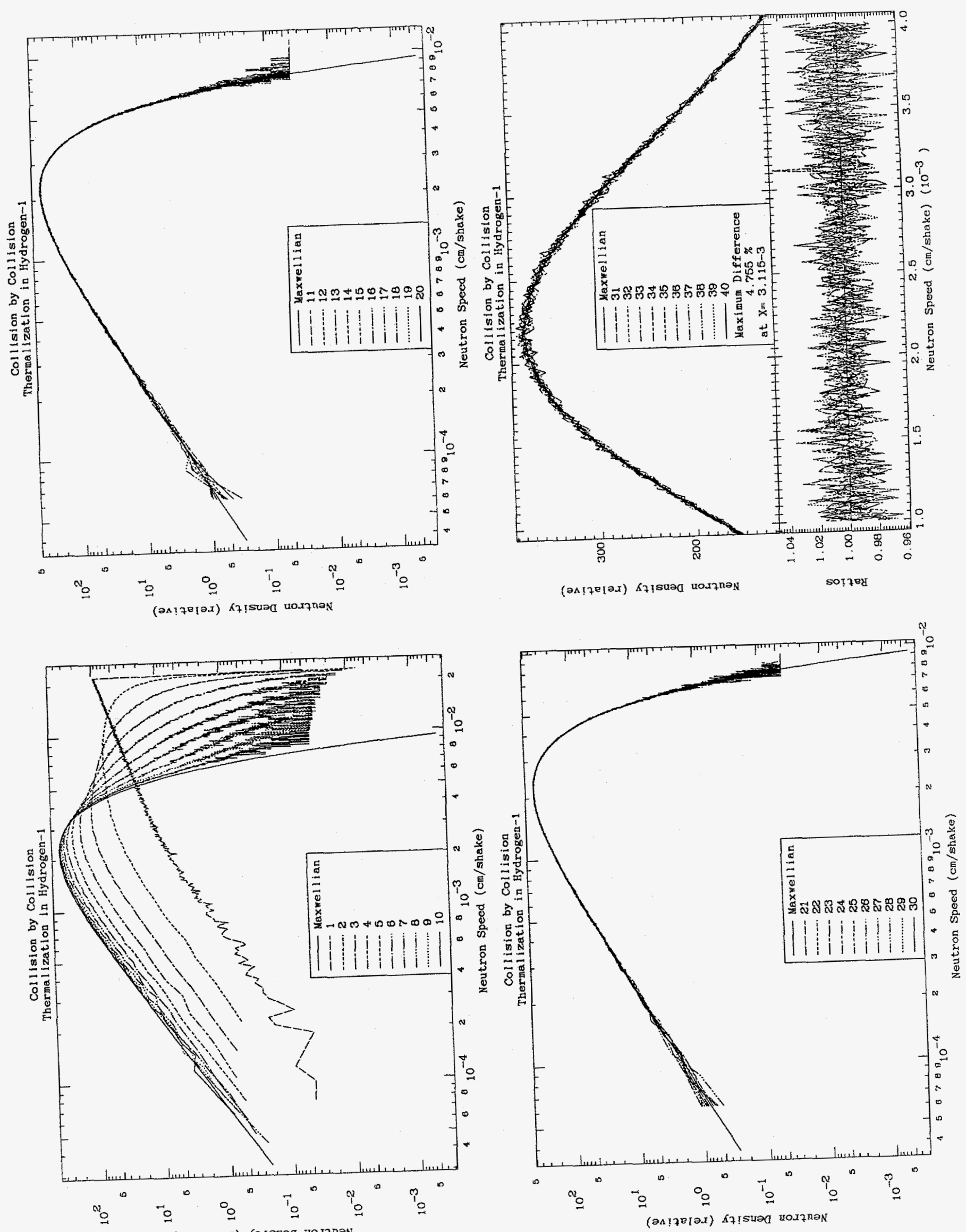

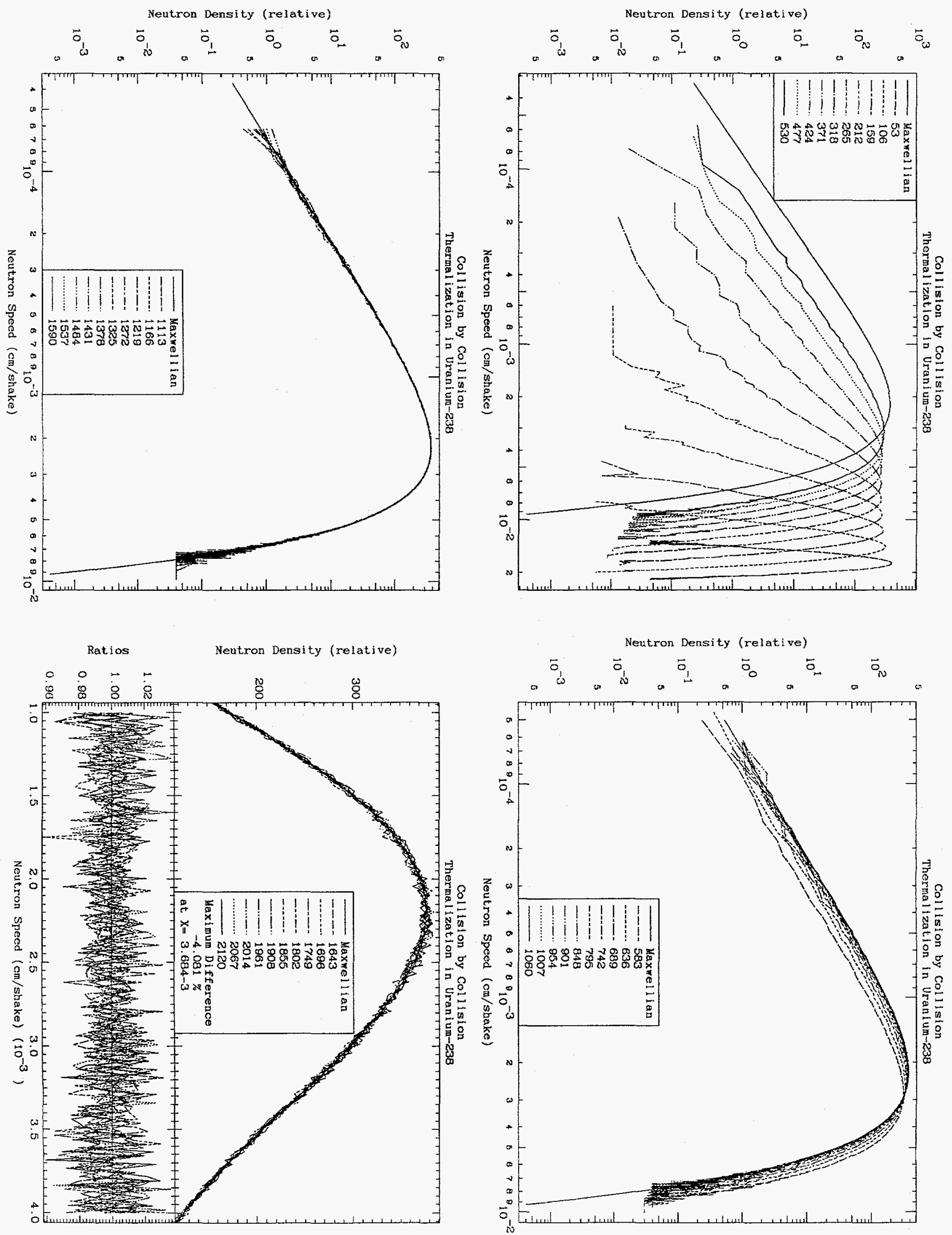

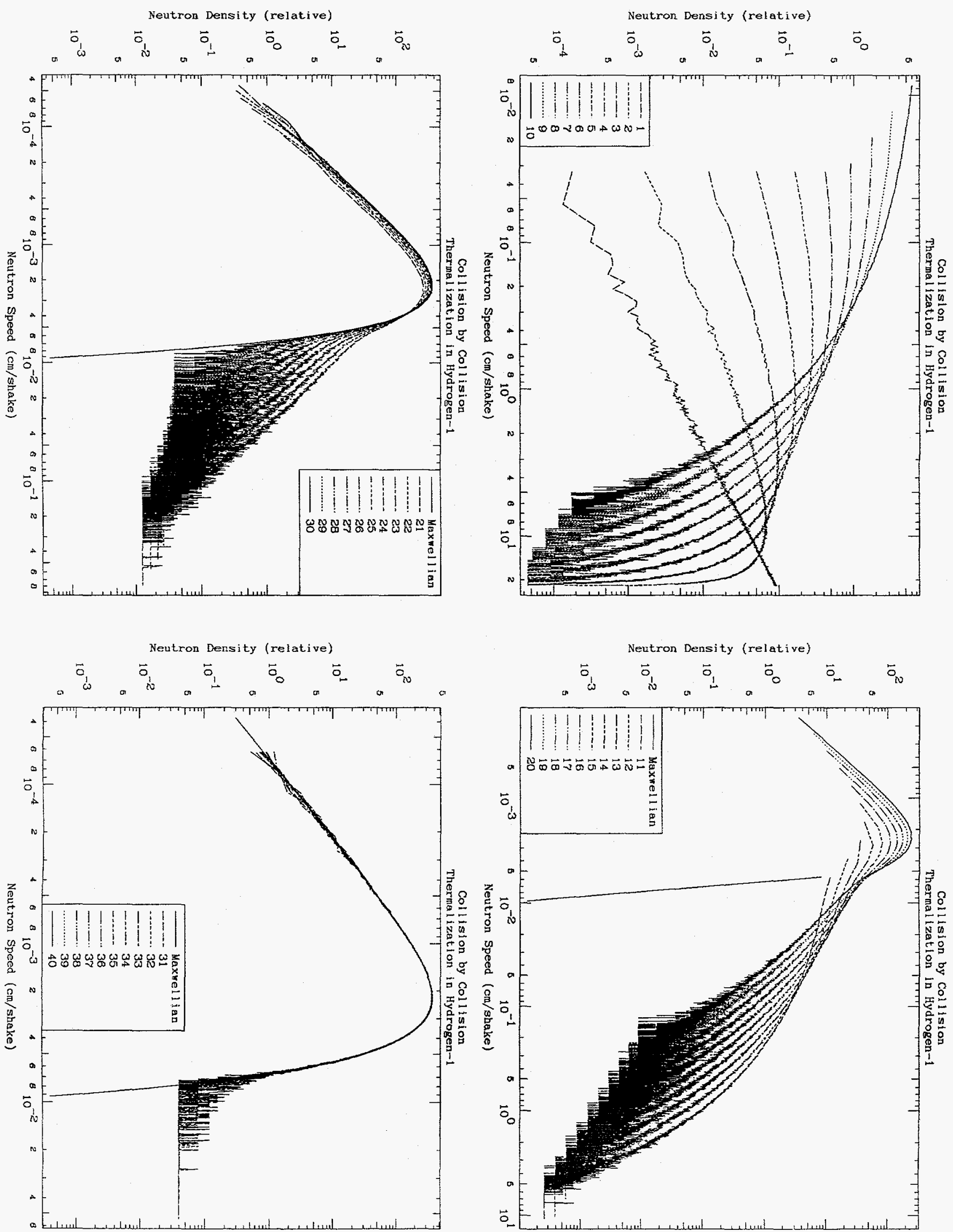